The Chittagong Univ. J. Sci. 40 : 111-136, 2018

\title{
Effect of Fukushima Accident on Tourists Based Coastal Belts of Chittagong City, Bangladesh
}

Hasan Murad $^{1}$, Md. Kowsar Alam ${ }^{1}$, Shyamal Ranjan Chakraborty ${ }^{1}$, A. K. M. Rezaur Rahman ${ }^{1}$, S. I. Bhuian ${ }^{2}$, Masud Kamal ${ }^{2}$, Akhi Das Gupta ${ }^{1}$, and Arun Kumar Deb ${ }^{1}$

${ }^{1}$ Department of Physics, University of Chittagong, Chittagong-4331, Bangladesh

${ }^{2}$ Atomic Energy Centre, Bangladesh Atomic Energy Commission, Chittagong, Bangladesh

*Corresponding author: <shyamal@cu.ac.bd>

\begin{abstract}
This research work was aimed to obtain the radioactive exposure of naturally occurring and anthropogenic radioactive materials in sediment samples from the coastal belts of Chittagong city due to the Fukushima nuclear accident. The activity concentration, absorbed outdoor and indoor dose rates, annual effective dose rates and the radiation hazard indices from these samples were calculated. The correlation coefficient and Chi-square value per degree of freedom were also determined. A strong correlation between radium equivalent activity and activity of ${ }^{232} \mathrm{Th}$ was obtained in the Bay of Bengal. However, a very poor correlation was observed for radium equivalent activity and activity of ${ }^{40} \mathrm{~K}$ in both the study areas. The Chi-square value per degree of freedom $\left(\chi_{R}^{2}\right)$ for ${ }^{226} \mathrm{Ra},{ }^{232} \mathrm{Th}$ and ${ }^{40} \mathrm{~K}$ were respectively 70.244, 77.168 and 1.87 for the Karnaphuli River samples and 38.091, 62.023 and 2.06 for the Bay of Bengal samples. No artificial radionuclide was detected in the samples measured from the study areas. No radiation threat level is obtained on the study areas due to the nuclear explosion at Fukushima.
\end{abstract}


112 Hasan Murad, Md. Kowsar Alam, Shyamal Ranjan Chakraborty, A. K. M. Rezaur Rahman, S. I. Bhuian, Masud Kamal, Akhi Das Gupta, and Arun Kumar Deb

Keywords: Fukushima accident; Radiation exposure; Correlation; Chi-square values.

চট্রাম নগর সংলগ্ন উপকূলীয় এলাকার পাললিক নমুনাসমূহে ফুকুশিমা পারমাণবিক দূর্ঘটনা জনিত কারণে প্রাকৃতিক ও কৃত্রিম ভাবে সৃষ্ট তেজস্ক্রিয় পদার্থ থেকে সম্পাত বের করার উদ্দেশ্যে এ গবেষণা কর্মটি সম্পাদন করা হয়েছিল। এ নমুনাসমূহে সক্রিয়তা ঘনত্ব, শোষিত গৃহস্থিত ও বহিরঙ্গন দাগহারসমূহ, বার্ষিক কার্যকর দাগহারসমূহ এবং বিকিরণ বিপদ সূচক হিসেব করা হয়েছিল। সহ-সম্বন্ধাঙ্ক এবং প্রতি স্বাধীনতার মাত্রায় কাই-বর্গ মানও বের করা হয়েছিল। বঙ্গোপসাগরে রেডিয়াম তুল্য সক্রিয়তা এবং ${ }^{232} \mathrm{Th}$-এর সক্রিয়তার মাঝে সুদৃঢ় সহ-সম্বন্ধ পাওয়া গিয়েছিল। সে যাই হোক, উভয় পর্র্যণা এলাকাতেই রেডিয়াম তুল্য সক্রিয়তা এবং ${ }^{40} \mathrm{~K}$ -এর সক্রিয়তার মাঝে খুব দূর্বল সহ-সম্বন্ধ পাওয়া গিয়েছিল। প্রতি স্বাধীনতার মাত্রায় কাই-বর্গ $\left(\chi_{R}^{2}\right)$ মান ${ }^{226} \mathrm{Ra},{ }^{232} \mathrm{Th}$ এবং ${ }^{40} \mathrm{~K}$-এর জন্য যথাক্রনে কর্ণফুলী নদীর নমুনাসমূহের জন্য ৭০.২৪৪, ৭৭.১৬৮ এবং ১.৮-৭ ও বঙ্গোপসাগরের নমুনাসমূহের জন্য ৩৮.০৯১, ৬২.০২৩ এবং ২.০৬ পাওয়া গিয়েছিল। পর্র্যেণা এলাকা সমূহের নমুনাগুলোতে কোন কৃত্রিম তেজস্ক্রিয় নিউক্লিয়ালের অস্তিত্ব পাওয়া যায়নি। ফুকুশিমায় পারমাণবিক বিস্ফোরণের জন্য পর্লেষণা এলাকা সমূহে কোন বিকিরণ ঝুঁকি পাওয়া যায়নি।

\section{Introduction}

Naturally occurring environmental radioactivity and the associated external exposure due to gamma radiation depend primarily on the geological and geographical conditions and appear at different levels in the soils of each region in the world [1]. All radionuclides release ionizing radiation that may cause cancer. 
Any amount of radiation dose may cause stochastic effect (e.g. cancer etc.). Uncontrolled radiation from any source is harmful to the occupational workers, public and environment. Since these radionuclides are not uniformly distributed, the knowledge of their distribution in soils plays an important role in radiation protection and measurement [2]. Also, the radioactivity of soils is essential for understanding changes in the natural background [3, 4]. For Bangladesh, tourism is an important economic activity. Important tourists' destinations and holiday resorts include beaches, river sides, parks and other historic places of interests. The Bay of Bengal (Patenga Sea Beach) and Karnaphuli river bank (Shah Amanat International Airport) are two important places for the people of the Chittagong City. The environmental quality of these places is an important attraction to large number of tourists and other holiday makers. But these two coastal places are linked to the Pacific Ocean through the Bay of Bengal. However, the wastes of the Fukushima nuclear accident were dumped naturally into the Pacific Ocean due to the effect of Tsunami. As a result, there could have some probabilities of increasing background radiation level of the natural and anthropogenic radionuclides present in the coastal belts of the present study areas. Among the environmental quality parameters, radiological hazard play a significant role in assessing the exposure of the public to natural radioactivity due to the presence of the uranium, thorium series and potassium-40. Sediment is mineral deposits formed through the weathering and erosion of rocks. These deposits found at different levels within the sand contain natural radionuclides that contribute to ionizing radiation exposure on earth [5]. The aim of the present study was categorized (1) to determine the specific activity 
114 Hasan Murad, Md. Kowsar Alam, Shyamal Ranjan Chakraborty, A. K. M. Rezaur Rahman, S. I. Bhuian, Masud Kamal, Akhi Das Gupta, and Arun Kumar Deb

of naturally occurring radionuclides ${ }^{226} \mathrm{Ra},{ }^{232} \mathrm{Th}$, and ${ }^{40} \mathrm{~K}$ and anthropogenic radionuclide ${ }^{137} \mathrm{Cs}$ and their corresponding radiological parameters in the sediment samples of the Karnaphuli River and the Bay of Bengal (Patenga sea beach) by using HPGe gamma ray spectrometry system, and (2) to obtain the correlation between Radium equivalent activity and the activity concentration of ${ }^{226} \mathrm{Ra},{ }^{232} \mathrm{Th}$, ${ }^{40} \mathrm{~K}$ and the Chi-square $\left(\chi_{R}^{2}\right)$ values [6] of the activity of ${ }^{226} \mathrm{Ra},{ }^{232} \mathrm{Th},{ }^{40} \mathrm{~K}$.

\section{Materials and Methods}

\subsection{Study Area}

Bay of Bengal (Patenga Sea beach) and the Karnaphuli River (in front of Shah Amanat International Airport) were the area of interest of the present study which are located at the metropolitan area of Chittagong city in Bangladesh. Patenga sea beach is situated about 22 kilometers away and to the west of Chittagong city. On the way to the beach, one passes the Shah Amanat International Airport.

\subsection{Sampling and Preparation}

A total of 20 sediment samples were collected from the study area in which 10 from Karnaphuli River bank (Shah Amanat International Airport) and 10 from the Bay of Bengal (Patenga sea beach). All the samples were collected using sampling grid system maintaining a distance of about $50 \mathrm{~m}$ from each other. About $1 \mathrm{~kg}$ of sample was collected from each location and each of the samples was preserved in sequentially numbered plastic packet. All the samples were transferred to the laboratory then air-dried initially. All the samples were crushed to fine powder using mortar and pastel after removing the bulk materials and then oven dried at 
$105^{\circ} \mathrm{C}$ and then homogenized by passing through a $1 \mathrm{~mm}$ mesh sieve. The homogenized samples were preserved into the cylindrical containers $(7.5 \mathrm{~cm}$ diameter and $3 \mathrm{~cm}$ height) and marked individually with identification parameters and then weighed. All the sample containers were sealed tightly with cap and wrapped with Teflon and thick vinyl tapes around their screw necks and finally air

tightened with polythene pack and stored for minimum four weeks prior to counting for allowing the establishment of secular equilibrium between the long lived ${ }^{226} \mathrm{Ra},{ }^{232} \mathrm{Th}$ and their decay products [7].

\subsection{Gamma Spectrometry}

\subsubsection{Detector Specification}

Gamma ray acquisitions of all the samples were done using a high-resolution ptype intrinsic High Purity Germanium (HPGe) coaxial detector mounted vertically and coupled to $16 \mathrm{~K}$ multichannel analyzer (Canberra). The detector was housed inside a lead shield to reduce the background radiation of the system. The experimental HPGe detector had a relative efficiency of $30 \%$ and a resolution of $1.78 \mathrm{keV}$ for the gamma emission of $1333 \mathrm{keV}$ of ${ }^{60} \mathrm{Co}$. The spectrums of the gamma-rays were analyzed using the Genie 2000 software associated with the detector.

\subsubsection{Efficiency and Specific Activity Measurement}

Prior to the analysis, energy and efficiency calibrations were performed in the energy range up to $2700 \mathrm{keV}$ to identify and quantify the radionuclides in the samples. The full-energy peak efficiency curve was plotted by measuring the reference samples IAEA/RGU-1 (U ore), IAEA/RGTh-1 (Th ore) and IAEA/RGK- 


\section{Hasan Murad, Md. Kowsar Alam, Shyamal Ranjan Chakraborty, A. K. M. Rezaur Rahman, S. I. Bhuian, Masud Kamal, Akhi Das Gupta, and Arun Kumar Deb}

1 (K sulphate) from which the counting efficiencies of the $\gamma$-ray peaks were measured [8]. The geometry of the counting samples was the same as that of the standard samples and the counting time for all the samples was $10,000 \mathrm{sec}$. The background count due to naturally occurring radionuclides in the environment around the detector was subtracted from each sample count. Background count was taken by a blank plastic container of the same geometry of the detector. The background spectra at least should be taken for 20,000 sec [9]. In the present study, the background spectra were taken for $20000 \mathrm{sec}$. The counting efficiency of the detector was calculated by using the following formula [10]:

$$
\% \text { Efficiency }=\frac{C P S \times 100}{\text { Activity } \times \text { Intensity }} \quad \text { or, } \varepsilon_{f}(\%)=\frac{C P S \times 100}{A_{C} \times I_{\gamma}}
$$

For determining the activity concentration of ${ }^{232} \mathrm{Th}$ the most gamma yielding energies of ${ }^{212} \mathrm{~Pb}(238.63 \mathrm{keV}),{ }^{212} \mathrm{Bi}(727.17 \mathrm{keV}),{ }^{208} \mathrm{Tl}(510.57 \mathrm{keV}, 583.19 \mathrm{keV}$ and $2614.53 \mathrm{keV})$ and ${ }^{228} \mathrm{Ac}(338.40 \mathrm{keV}, 911.07$ and $969.11 \mathrm{keV})$ were used. In case of the secular equilibrium, the gamma ray energies of ${ }^{214} \mathrm{~Pb}(295.21 \mathrm{keV})$ and ${ }^{214} \mathrm{Bi}(1764.49 \mathrm{keV})$ and ${ }^{214} \mathrm{~Pb}(351.92 \mathrm{keV})$ and ${ }^{214} \mathrm{Bi}(609.31 \mathrm{keV})$ were used to calculate the specific activity of ${ }^{226} \mathrm{Ra}$. The ${ }^{40} \mathrm{~K}$ and ${ }^{137} \mathrm{Cs}$ radionuclides were measured from their respective $\gamma$-ray energies $1460.75 \mathrm{keV}$ and $661.66 \mathrm{keV}$ respectively [11]. The activities of the natural radionuclide presented in the sediment samples were calculated by using the following formula [12, 13]:

$$
\text { Activity }=\frac{C P S \times 100 \times 1000}{\varepsilon_{f}(\%) \times I_{\gamma} \times w_{s}(g m)}
$$

Where, CPS $=$ Net counts per second (i.e., CPS for sample - CPS for background) 


$$
\begin{aligned}
& \varepsilon_{f}=\text { Counting gamma energy efficiency of the detector in percentage. } \\
& I_{\gamma}=\text { Intensity of the gamma ray or gamma yielding. }
\end{aligned}
$$

The results are expressed with the confidence limit of $\pm 1 \sigma$.

\subsubsection{Calculation of Radiological Parameters}

(a) The formula for calculating the outdoor absorbed dose rate in air at $1 \mathrm{~m}$ above the ground surface (in $n G y \cdot h^{-1}$ ) using the conversion factors given in the UNSCEAR 1988 [14] report is

$$
\mathrm{D}_{\text {outdoor }}=\left(0.427 \mathrm{C}_{\mathrm{Ra}}+0.66 \mathrm{C}_{\mathrm{Th}}+0.0432 \mathrm{C}_{\mathrm{K}}\right)
$$

Where $\mathrm{C}_{\mathrm{Ra}}, \mathrm{C}_{\mathrm{Th}}$ and $\mathrm{C}_{\mathrm{K}}$ are average activity concentrations of ${ }^{226} \mathrm{Ra},{ }^{232} \mathrm{Th}$, and ${ }^{40} \mathrm{~K}$ respectively in the sediment samples.

(b) The indoor contribution is assumed to be 1.2 times higher than the outdoor dose [14]

$$
\mathrm{D}_{\text {indoor }}=\mathrm{D}_{\text {outdoor }} \times 1.2\left(\mathrm{nGy} \cdot \mathrm{h}^{-1}\right)
$$

(c) The annual effective dose equivalent $\mathrm{D}_{\text {eff }}$ from outdoor terrestrial gamma radiation is [15]

$$
\mathrm{D}_{\text {eff }}=\text { Outdoor dose }\left(\mathrm{nGy} \cdot \mathrm{h}^{-1}\right) \times 0.7\left(\mathrm{~Sv}_{\mathrm{G}} \mathrm{G}^{-1}\right) \times 8,760\left(\mathrm{~h} \cdot \mathrm{y}^{-1}\right) \times 0.2
$$

Where 0.2 is the outdoor occupancy factor and $0.7 \mathrm{~Sv} \mathrm{~Gy}^{-1}$ is the quotient of effective dose equivalent rate to absorbed dose rate in air. For indoor exposure, using an occupancy factor of 0.8 , the annual effective dose equivalent is

$$
\mathrm{D}_{\text {eff }}=\text { Indoor dose }\left(\mathrm{nGy} \cdot \mathrm{h}^{-1}\right) \times 0.7\left(\mathrm{~Sv}_{\mathrm{Gy}}{ }^{-1}\right) \times 8,760\left(\mathrm{~h} . \mathrm{y}^{-1}\right) \times 0.8
$$

(d) The total annual effective dose equivalent from terrestrial radiation is the sum of outdoor and indoor annual effective dose equivalent. 
118 Hasan Murad, Md. Kowsar Alam, Shyamal Ranjan Chakraborty, A. K. M. Rezaur Rahman, S. I. Bhuian, Masud Kamal, Akhi Das Gupta, and Arun Kumar Deb

(e) The sediment is used for different purposes so the formula for calculating the external radiation hazard, $\mathrm{H}_{\mathrm{ext}}$ and internal radiation hazard, $\mathrm{H}_{\mathrm{int}}$ are as follows [16].

$$
\begin{aligned}
& \mathrm{H}_{\mathrm{ext}}=\mathrm{A}_{\mathrm{Ra}} / 370+\mathrm{A}_{\mathrm{Th}} / 259+\mathrm{A}_{\mathrm{K}} / 4810 \\
& \mathrm{H}_{\mathrm{int}}=\mathrm{A}_{\mathrm{Ra}} / 185+\mathrm{A}_{\mathrm{Th}} / 259+\mathrm{A}_{\mathrm{K}} / 4810
\end{aligned}
$$

The numerical quantities of equations (5) and (6) are in units of Bq. $\mathrm{kg}^{-1}$ and $\mathrm{A}_{\mathrm{Ra}}$, $\mathrm{A}_{\mathrm{Th}}$ and $\mathrm{A}_{\mathrm{K}}$ are the activity concentration of ${ }^{226} \mathrm{Ra},{ }^{232} \mathrm{Th}$ and ${ }^{40} \mathrm{~K}$ respectively.

(f) The formula for calculating the radium equivalent activities, $\mathrm{Ra}_{\mathrm{eq}}$ and the representative level index values, $\mathrm{I}_{\gamma}$ are as follows [16]

$$
\begin{aligned}
& \mathrm{Ra}_{\mathrm{eq}}=\mathrm{A}_{\mathrm{Ra}}+1.43 \mathrm{~A}_{\mathrm{Th}}+0.077 \mathrm{~A}_{\mathrm{K}^{--}} \\
& \mathrm{I}_{\gamma}=\left(\mathrm{C}_{\mathrm{Ra}} / 150+\mathrm{C}_{\mathrm{Th}} / 100+\mathrm{C}_{\mathrm{K}} / 1500\right)
\end{aligned}
$$

Where $A_{R a}, A_{T h}$ and $A_{K}$ in equation (7) and $C_{R a}, C_{T h}$ and $C_{K}$ in equation (8) are activity concentrations of ${ }^{226} \mathrm{Ra},{ }^{232} \mathrm{Th}$, and ${ }^{40} \mathrm{~K}$ respectively in the sediment samples respectively.

\section{Results and Discussion}

\subsection{Activity concentration of ${ }^{226} \mathrm{Ra},{ }^{232} \mathrm{Th}$ and ${ }^{40} \mathrm{~K}$}

The mean activity concentrations of ${ }^{226} \mathrm{Ra}$ was found to be $38.33 \pm 0.64 \& 48.89 \pm$ $0.77 \mathrm{~Bq} \cdot \mathrm{kg}^{-1}$ and ranged from $18.68 \pm 2.22$ to $66.94 \pm 1.86$ and $28.56 \pm 2.27$ to $73.61 \pm 2.30 \mathrm{~Bq} \cdot \mathrm{kg}^{-1}$ for the Karnaphuli River and the Bay of Bengal respectively. The mean values of specific activities of these radionuclides for both the study areas were higher than those of the world average [1].

The activity concentrations of ${ }^{223} \mathrm{Th}$ had been found to be ranged from $19.36 \pm 1.35$ to $61.56 \pm 1.69$ and $29.17 \pm 1.78$ to $69.84 \pm 1.94 \mathrm{~Bq} \cdot \mathrm{kg}^{-1}$ with a mean value of 
$35.18 \pm 0.49 \& 50.12 \pm 0.61$ Bq. $\mathrm{kg}^{-1}$ for the Karnaphuli River and the Bay of Bengal respectively. The mean activity concentration of ${ }^{232} \mathrm{Th}$ in the Karnaphuli River samples was lower than that of the world average [1]. However, the mean value of concentration for Bay of Bengal was higher than that of the world average value.

The activity concentrations of ${ }^{40} \mathrm{~K}$ was found to be ranged from $290.01 \pm 27.23$ to $521.97 \pm 58.08$ and $314.61 \pm 93.77$ to $677.42 \pm 105.86 \mathrm{~Bq} \cdot \mathrm{kg}^{-1}$ with the mean value of $355.31 \pm 19.97$ and $478.64 \pm 31.35$ Bq. $\mathrm{kg}^{-1}$ for the Karnaphuli River and the Bay of Bengal respectively. The mean concentration of ${ }^{40} \mathrm{~K}$ in the Karnaphuli River samples was lower whereas for the Bay of Bengal which was higher than that of the world average. The comparison of the mean specific activity concentrations of ${ }^{226} \mathrm{Ra},{ }^{232} \mathrm{Th}$ and ${ }^{40} \mathrm{~K}$ for both the study areas are given in Table 1 and represented in Figure 1. However, no artificial radionuclide like ${ }^{137} \mathrm{Cs}$ had been detected in the samples of present study area. The comparisons of these radionuclides for the Karnaphuli River and the Bay of Bengal with some other studies of the world are given in Tables 2 and 3, respectively. 
120 Hasan Murad, Md. Kowsar Alam, Shyamal Ranjan Chakraborty, A. K. M. Rezaur Rahman, S. I. Bhuian, Masud Kamal, Akhi Das Gupta, and Arun Kumar Deb

Table 1: Comparison of mean activity concentration (in Bq. $\mathrm{kg}^{-1}$ ) of the Karnaphuli River, the Bay of Bengal and UNSCEAR 2000.

\begin{tabular}{lcccc}
\hline \multicolumn{1}{c}{ Reference } & ${ }^{226} \mathbf{R a}$ & ${ }^{232} \mathbf{T h}$ & ${ }^{40} \mathbf{K}$ & ${ }^{137} \mathbf{C s}$ \\
\hline Karnaphuli river & $38.33 \pm 0.64$ & $35.18 \pm 0.49$ & $355.31 \pm 19.97$ & ND \\
Bay of Bengal & $48.89 \pm 0.77$ & $50.12 \pm 0.06$ & $478.64 \pm 31.35$ & ND \\
1 & 33 & 45 & 420 & - \\
\hline
\end{tabular}

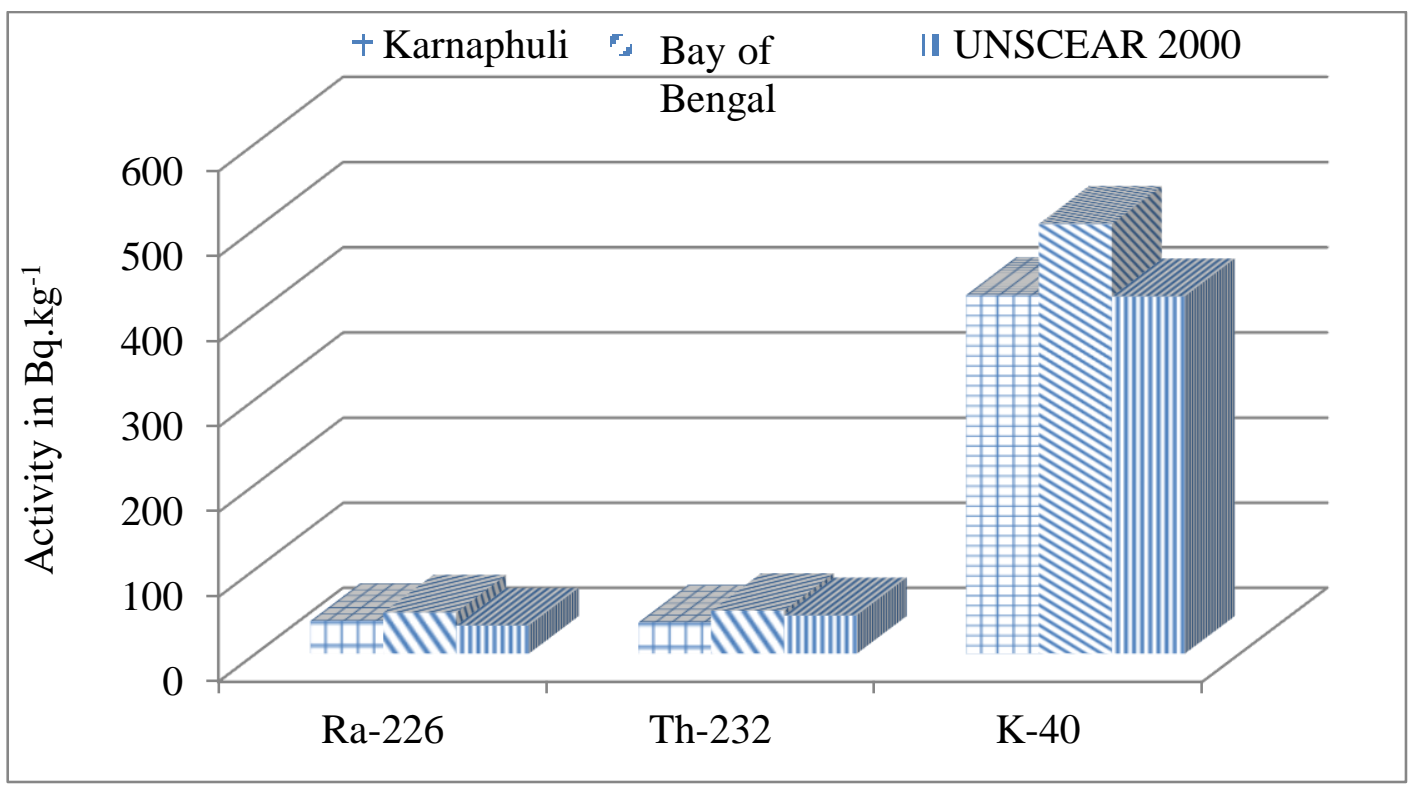

Figure 1: Comparison of average activities of the Karnaphuli River, the Bay of Bengal and UNSCEAR 2000. 
Table 2: Comparison of the mean activity concentration (in Bq.kg-1) of ${ }^{226} \mathrm{Ra},{ }^{232} \mathrm{Th}$ and ${ }^{40} \mathrm{~K}$ of the Karnaphuli River with some other rivers of the world and worldwide values.

\begin{tabular}{ccccc}
\hline Place & ${ }^{226} \mathrm{Ra}$ & ${ }^{232} \mathrm{Th}$ & ${ }^{40} \mathrm{~K}$ & Reference \\
\hline Safaga, Egypt & 25.3 & 21.4 & 618 & 17 \\
Xianyang, China & 31.1 & 44.9 & 776 & 17 \\
Baoji, China & 22.1 & 39 & 859 & 17 \\
Brazil & 7810 & 17770 & 2660 & 17 \\
SaudiArabia & $4.35 \pm 0.028$ & $3.3 \pm 0.033$ & $71.74 \pm 7.21$ & 18 \\
Gediz, Turky & $35.61-62.64$ & $7.40-38.53$ & $240.40-403.09$ & 19 \\
River Agbo, Nigeriain & $9.40 \pm 0.41$ & - & $383.28 \pm 9.24$ & 20 \\
River Afelumo, Nigeria & $52.71 \pm 0.97$ & - & $495.12 \pm 10.50$ & 20 \\
River Oyinmo, Nigeria & $16.56 \pm 0.05$ & - & $514.85 \pm 14.27$ & 20 \\
Wei River & $10.4-39.9$ & $15.3-54.8$ & $514.8-1175.5$ & 21 \\
Cauvery River, & $5.31 \pm 0.4$ & $34.04 \pm 1.4$ & $401.11 \pm 24.3$ & 22 \\
Tamilnadu, India & & & & 23 \\
Nile River, Egypt & $52 \pm 7.3$ & $76.2 \pm 6.2$ & $351.9 \pm 17.6$ & (Present \\
Karnaphuli River & $38.33 \pm 0.64$ & $35.18 \pm 0.49$ & $355.31 \pm 19.97$ & Study) \\
World average & 33 & 45 & 420 & 1 \\
\hline
\end{tabular}


122 Hasan Murad, Md. Kowsar Alam, Shyamal Ranjan Chakraborty, A. K. M. Rezaur Rahman, S. I. Bhuian, Masud Kamal, Akhi Das Gupta, and Arun Kumar Deb

Table 3: Comparison of the mean activity concentration (in Bq.kg ${ }^{-1}$ ) of ${ }^{226} \mathrm{Ra},{ }^{232} \mathrm{Th}$ and ${ }^{40} \mathrm{~K}$ of the Bay of Bengal with some other Beaches of the world and worldwide values.

\begin{tabular}{|c|c|c|c|c|}
\hline Place & ${ }^{226} \mathrm{Ra}$ & ${ }^{232} \mathrm{Th}$ & ${ }^{40} \mathrm{~K}$ & Reference \\
\hline Persian Gulf, Iran & 35 & 26 & 395 & 17 \\
\hline $\begin{array}{c}\text { Coast of Greater } \\
\text { Accra }\end{array}$ & 22.04 & 108.60 & 29.78 & 17 \\
\hline Northwest Libya & $7.5(4-13.5)$ & $4.2(2.8-6.7)$ & $27.5(19-39.6)$ & 24 \\
\hline $\begin{array}{c}\text { Safaga, Red Sea; } \\
\text { Egypt }\end{array}$ & 28.82 & 14.03 & 558.39 & 22 \\
\hline $\begin{array}{c}\text { Idku coast, Behara; } \\
\text { Egypt }\end{array}$ & 13.08 & 13.97 & 345.97 & 25 \\
\hline $\begin{array}{c}\text { Chalatat and the } \\
\text { Samila beaches, } \\
\text { Thailand }\end{array}$ & $41 \pm 5$ & $64 \pm 7$ & $248 \pm 44$ & 26 \\
\hline $\begin{array}{c}\text { Kuakata, } \\
\text { Bangladesh }\end{array}$ & $29.48 \pm 3.85$ & $93.72 \pm 15.62$ & $551.24 \pm 109.95$ & 27 \\
\hline Beaches of Ghana & 31.4 & 42.6 & 109.5 & 28 \\
\hline Japan & 33 & 28 & 310 & 1 \\
\hline USA & 40 & 35 & 370 & 1 \\
\hline Poland & 26 & 21 & 410 & 1 \\
\hline Greece & 25 & 21 & 360 & 1 \\
\hline Spain & 32 & 33 & 470 & 1 \\
\hline Hong Kong SAR & 59 & 95 & 530 & 1 \\
\hline Denmark & 17 & 19 & 460 & 1 \\
\hline Bay of Bengal & $48.89 \pm 0.77$ & $50.12 \pm 0.61$ & $478.64 \pm 31.35$ & $\begin{array}{c}\text { (Present } \\
\text { Study) }\end{array}$ \\
\hline World Average & 33 & 45 & 420 & 1 \\
\hline
\end{tabular}




\subsection{Radium Equivalent Activity $\mathbf{R a}_{\mathrm{eq}}$}

The mean value of the Radium equivalent activity had been found to be $107.23 \pm$ 6.89 and $150.47 \pm 10.42 \mathrm{~Bq} \cdot \mathrm{kg}^{-1}$ with a range of $85.26 \pm 21.55$ to $174.51 \pm 20.51$ and $109.74 \pm 23.34$ to $215.41 \pm 23.73 \mathrm{~Bq} \cdot \mathrm{kg}^{-1}$ for the Karnaphuli River and the Bay of Bengal respectively. The obtained mean value is lower for the Karnaphuli River but higher for the Bay of Bengal than that of the world average. The comparison of $\mathrm{Ra}_{\mathrm{eq}}$ for both the study areas with the world average is shown Figure 2 .

\subsection{Gamma Dose Rates}

The calculated values of indoor gamma dose rates were ranged from $43.68 \pm 11.33$ to $98.49 \pm 20.63$ and $68.69 \pm 13.38$ to $119.62 \pm 19.78 \mathrm{nGy} \cdot \mathrm{h}^{-1}$ with an average of $61.93 \pm 3.97$ and $86.83 \pm 6.00 \mathrm{nGy} \cdot \mathrm{h}^{-1}$ for the Karnaphuli River and the Bay of Bengal respectively. Whereas, the calculated mean value of outdoor gamma dose rate was found to be $51.61 \pm 3.31$ and $72.36 \pm 5.00 \mathrm{nGy} \cdot \mathrm{h}^{-1}$ with a range from $36.39 \pm 9.44$ to $82.08 \pm 20.63$ and $57.25 \pm 11.15$ to $99.68 \pm 16.48 \mathrm{nGy} \cdot \mathrm{h}^{-1}$ respectively, for the Karnaphuli River and the Bay of Bengal.

The external outdoor gamma dose rates were also measured by using $\beta-\gamma$ survey meter of model LUDLUM 44-9. The measured outdoor dose rate for both the study areas was higher than that of the calculated value. The comparative data of the measured outdoor dose rate, calculated outdoor dose rate and world average is illustrated in Figure 3. 
124 Hasan Murad, Md. Kowsar Alam, Shyamal Ranjan Chakraborty, A. K. M. Rezaur Rahman, S. I. Bhuian, Masud Kamal, Akhi Das Gupta, and Arun Kumar Deb

\subsection{Representative Level Index}

The value of representative level index found to be ranged from $0.55 \pm 0.15$ to 1.26 \pm 0.26 and $0.88 \pm 0.17$ to $1.53 \pm 0.25 \mathrm{~Bq} \cdot \mathrm{kg}^{-1}$ with a mean value of $0.79 \pm 0.05$ and $1.11 \pm 0.08 \mathrm{~Bq} \cdot \mathrm{kg}^{-1}$ for the Karnaphuli River and the Bay of Bengal respectively. The obtained mean value is lower for the Karnaphuli River than that of the world average value whereas this value is higher for the Bay of Bengal.

\subsection{Radiation Hazard Indices}

The external hazard index were ranged from $0.20 \pm 0.05$ to $0.47 \pm 0.11$ and $0.31 \pm$ 0.06 to $0.56 \pm 0.09$ with an average of $0.28 \pm 0.02$ and $0.41 \pm 0.03$ for the Karnaphuli river and the Bay of Bengal respectively. However, the mean value of internal hazard index had been found to be $0.37 \pm 0.03$ and $0.52 \pm 0.04$ with a range of $0.25 \pm 0.07$ to $0.64 \pm 0.15$ and $0.39 \pm 0.08$ to $0.73 \pm 0.15$ for the Karnaphuli River and the Bay of Bengal respectively. The radiation hazard index for both the study areas have been found to be lower than that of the safety limit suggested by UNSCEAR 2000 [1]. So, the tourists and people of all occupation working there is free from the exposure of radiation danger for both the study areas. 


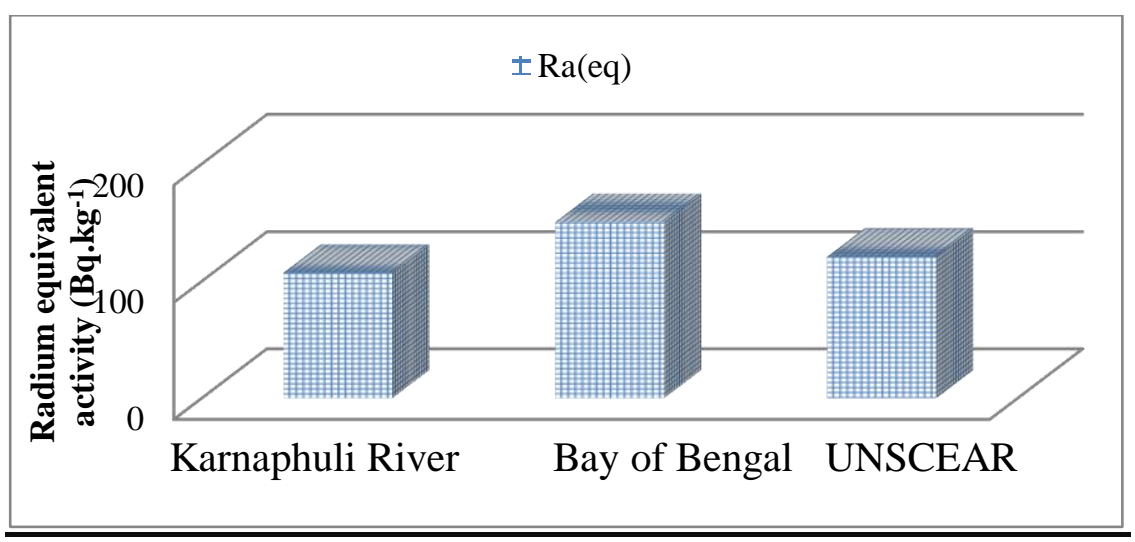

Figure 2: Comparison of Radium Equivalent Activities.

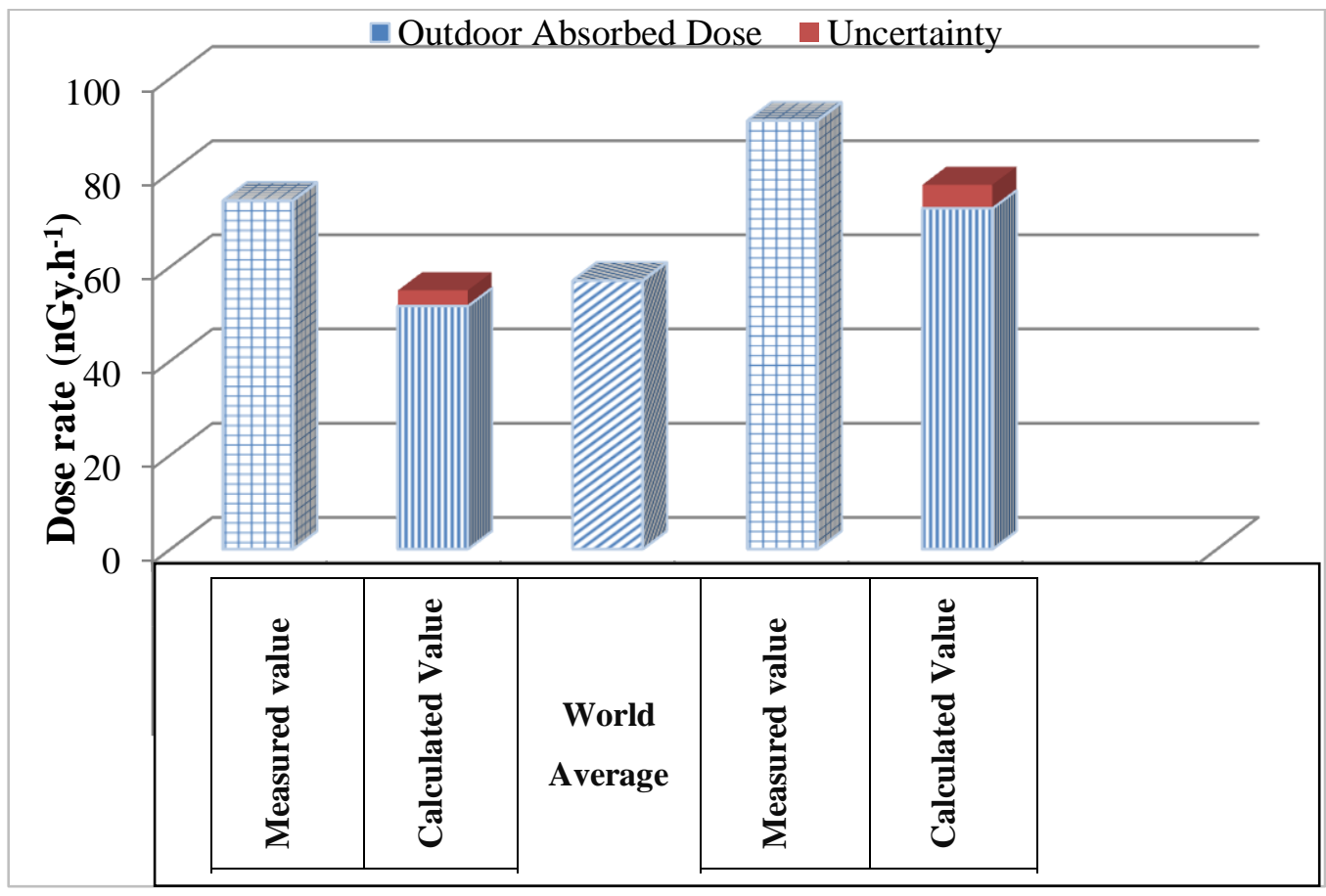

Figure 3: Comparison of Outdoor absorbed gamma dose rate of both study areas and the world average. 
Hasan Murad, Md. Kowsar Alam, Shyamal Ranjan Chakraborty, A. K. M. Rezaur Rahman, S. I. Bhuian, Masud Kamal, Akhi Das Gupta, and Arun Kumar Deb

\subsection{Annual and Total Annual Effective Dose Equivalents:}

The mean value of outdoor annual effective dose equivalent had been found to be $0.06 \pm 0.004$ and $0.09 \pm 0.006 \mathrm{mSv} \cdot \mathrm{y}^{-1}$ with the range of $0.04 \pm 0.01$ to $0.10 \pm$ 0.021 and $0.07 \pm 0.01$ to $0.12 \pm 0.02 \mathrm{mSv} \cdot \mathrm{y}^{-1}$ for the Karnaphuli River and the Bay of Bengal respectively. However, the indoor annual effective dose equivalent found to be ranged from $0.21 \pm 0.06$ to $0.48 \pm 0.10$ and $0.34 \pm 0.07$ to $0.59 \pm 0.09 \mathrm{mSv}_{\mathrm{y}} \mathrm{y}^{-1}$ with an average of $0.30 \pm 0.02$ and $0.43 \pm 0.03 \mathrm{mSv}^{-1} \mathrm{y}^{-1}$ for the Karnaphuli River and the Bay of Bengal respectively.

The total annual effective dose equivalent was found to be ranged from $0.04 \pm 0.01$ to $0.10 \pm 0.02$ and $0.07 \pm 0.01$ to $0.12 \pm 0.02 \mathrm{mSv} \cdot \mathrm{y}^{-1}$ with an average of $0.37 \pm$ 0.03 and $0.52 \pm 0.05$ mSv. $\mathrm{y}^{-1}$ for the Karnaphuli River and the Bay of Bengal respectively. The comparisons of the radiological parameters of the present research work with some other countries of the world are given in Table 4 and 5. 
Table 4: Comparison of radiological parameters with some other river sediments of the world.

\begin{tabular}{|c|c|c|c|c|c|c|}
\hline Place & $\begin{array}{c}\text { Radium } \\
\text { Equivalent } \\
\text { activity, } \\
\mathbf{R a}_{\text {eq }} \\
\left(\mathbf{B q}^{-1} \mathbf{k g}^{-1}\right)\end{array}$ & $\begin{array}{c}\text { Representative } \\
\text { Gamma Index, } \\
\quad \text { I } \gamma \\
\left(\mathbf{B q} \cdot \mathbf{k g}^{-\mathbf{1}}\right)\end{array}$ & $\begin{array}{c}\text { External } \\
\text { Radiation } \\
\text { Hazard } \\
\text { Index, } \\
\text { Hext }_{\text {ext }}\end{array}$ & $\begin{array}{c}\text { Total } \\
\text { absorbed } \\
\text { dose rate, D } \\
\left(\mathrm{nGy} \cdot \mathrm{h}^{-1}\right)\end{array}$ & $\begin{array}{c}\text { Annual } \\
\text { effective } \\
\text { dose, } \mathbf{H} \\
\left(\mathbf{m S v}_{\mathbf{m}} \mathbf{y}^{-1}\right)\end{array}$ & Reference \\
\hline $\begin{array}{c}\text { Safaga, } \\
\text { Egypt }\end{array}$ & $<370$ & 1.3 & - & 50.9 & - & 17 \\
\hline China & 155 & 1.17 & 0.42 & 73.9 & 0.091 & 17 \\
\hline Baoji, China & 144 & - & 039 & 69.6 & 0.085 & 17 \\
\hline Brazil & 25800 & 230 & 70 & 14450 & 17.70 & 17 \\
\hline Saudi Arabia & 20.16 & 0.149 & & 9.54 & 0.0115 & 18 \\
\hline River Agbo & 84.64 & - & - & 41.74 & - & 20 \\
\hline $\begin{array}{c}\text { River } \\
\text { Afelumo }\end{array}$ & 512.46 & - & - & 243.25 & - & 20 \\
\hline $\begin{array}{l}\text { River } \\
\text { Oyinmo }\end{array}$ & 113.42 & - & - & 55.79 & - & 20 \\
\hline China & $<370$ & $\approx 1$ & $<1$ & 68.8 & 0.079 & 23 \\
\hline $\begin{array}{c}\text { Cauvery } \\
\text { River, TN, } \\
\text { India }\end{array}$ & - & - & - & $40.73 \pm 1.8$ & 0.25 & 22 \\
\hline USA & 119 & - & - & 56 & - & 1 \\
\hline $\begin{array}{l}\text { Karnaphuli } \\
\text { River }\end{array}$ & $\begin{array}{c}107.23 \pm \\
6.89\end{array}$ & $0.79 \pm 0.05$ & $0.29 \pm 0.02$ & $51.61 \pm 3.31$ & $0.06 \pm 0.00$ & $\begin{array}{l}\text { (Present } \\
\text { Study) }\end{array}$ \\
\hline
\end{tabular}


128 Hasan Murad, Md. Kowsar Alam, Shyamal Ranjan Chakraborty, A. K. M. Rezaur Rahman, S. I. Bhuian, Masud Kamal, Akhi Das Gupta, and Arun Kumar Deb

Table 5: Comparison of radiological parameters with some other beaches of the world.

\begin{tabular}{|c|c|c|c|c|c|c|}
\hline Place & $\begin{array}{c}\text { Radium } \\
\text { Equivalent } \\
\text { activity, } \\
\mathrm{Ra}_{\mathrm{eq}}\left(\mathrm{Bq} \cdot \mathrm{Bg}^{-}\right. \\
1 \text { ) }\end{array}$ & $\begin{array}{c}\text { Representative } \\
\text { Gamma Index, } \\
\text { I } \gamma \\
\left(\mathrm{Bq} \cdot \mathrm{kg}^{-1}\right)\end{array}$ & $\begin{array}{c}\text { External } \\
\text { Radiation } \\
\text { Hazard } \\
\text { Index, } \mathrm{H}_{\mathrm{ext}}\end{array}$ & $\begin{array}{c}\text { Total } \\
\text { absorbed } \\
\text { dose rate, D } \\
\left(\mathrm{nGy} \cdot \mathrm{h}^{-1}\right)\end{array}$ & $\begin{array}{c}\text { Annual } \\
\text { effective } \\
\text { dose, } \mathrm{H} \\
\left(\mathrm{mSv} \cdot \mathrm{y}^{-1}\right)\end{array}$ & Reference \\
\hline $\begin{array}{l}\text { Persian Gulf, } \\
\text { Iran }\end{array}$ & $<370$ & - & $<1$ & 37 & - & 17 \\
\hline $\begin{array}{c}\text { Coast of } \\
\text { Greater } \\
\text { Accra }\end{array}$ & 9 & 0.48 & - & 77.02 & 0.09 & 17 \\
\hline $\begin{array}{l}\text { Northwest } \\
\text { Libya }\end{array}$ & - & - & - & $4.4 \pm 1.3$ & $\begin{array}{c}0.0054 \pm 0 \\
.0016\end{array}$ & 24 \\
\hline $\begin{array}{c}\text { Safaga, Red } \\
\text { Sea; Egypt }\end{array}$ & 370 & 1 & $\leq 1$ & 59 & - & 22 \\
\hline $\begin{array}{l}\text { Idku coast, } \\
\text { Behara; } \\
\text { Egypt }\end{array}$ & 57.67 & 0.4575 & 0.16122 & 29.71 & 0.03645 & 25 \\
\hline $\begin{array}{l}\text { Chalatat and } \\
\text { the Samila } \\
\text { beaches, } \\
\text { Thailand }\end{array}$ & $152 \pm 18$ & - & - & $69 \pm 8$ & - & 26 \\
\hline Ghana & 101.0 & 0.71 & & 54.08 & 0.066 & 28 \\
\hline $\begin{array}{l}\text { Kuakata, } \\
\text { Bangladesh }\end{array}$ & $\begin{array}{c}98.81 \\
\text { To } \\
271.17\end{array}$ & 0.8 to 3.75 & - & 98.33 & - & 27 \\
\hline USA & 119 & - & - & 56 & - & 1 \\
\hline China & 125 & - & - & 59 & - & 1 \\
\hline $\begin{array}{c}\text { Hong Kong } \\
\text { SAR }\end{array}$ & 236 & - & - & 108 & - & 1 \\
\hline Japan & 97 & - & - & 45 & - & 1 \\
\hline Denmark & 80 & - & - & 39 & - & 1 \\
\hline Switzerland & 104 & - & - & 49 & - & 1 \\
\hline Poland & 88 & - & - & 42 & - & 1 \\
\hline Greece & 83 & - & - & 40 & - & 1 \\
\hline Spain & 115 & - & - & 55 & - & 1 \\
\hline $\begin{array}{l}\text { Bay of } \\
\text { Bengal }\end{array}$ & $\begin{array}{c}150.47 \pm \\
10.42\end{array}$ & $1.11 \pm 0.08$ & $\begin{array}{c}0.41 \pm \\
0.03\end{array}$ & $72.36 \pm 5.00$ & $\begin{array}{c}0.52 \pm \\
0.05\end{array}$ & $\begin{array}{c}\text { (Present } \\
\text { Study) }\end{array}$ \\
\hline
\end{tabular}




\subsection{Correlation}

In the present study, a high degree of correlation between radium equivalent activities and ${ }^{226} \mathrm{Ra}$ activities was obtained for both the study areas which are represented in Figure 4. The dependence is higher for the Bay of Bengal samples than that of Karnaphuli River samples. A very high degree of correlation ( $\mathrm{r}=$ 0.9551 ) had been found to exist between the radium equivalent activities and ${ }^{232} \mathrm{Th}$ activities for the Bay of Bengal but a high degree of correlation $(r=0.8361)$ was found for the Karnaphuli River samples which is shown in Figure 5. This indicates a strong dependence of $\mathrm{Ra}_{\mathrm{eq}}$ on ${ }^{232} \mathrm{Th}$ concentration for both the study areas. However, the correlation between $\mathrm{Ra}_{\mathrm{eq}}$ and ${ }^{40} \mathrm{~K}$ for both the study areas had been found to be very poor $(r=0.0011$ and 0.0332 for the Bay of Bengal and the Karnaphuli river respectively). The poor correlation between radium equivalent activities and ${ }^{40} \mathrm{~K}$ in both the study areas indicates that $\mathrm{Ra}_{\mathrm{eq}}$ varied slightly on the presence of ${ }^{40} \mathrm{~K}$ which is given in Figure 6. It is obvious that all these correlations were positive as evident from the formula of radium equivalent activity. 
130 Hasan Murad, Md. Kowsar Alam, Shyamal Ranjan Chakraborty, A. K. M. Rezaur Rahman, S. I. Bhuian, Masud Kamal, Akhi Das Gupta, and Arun Kumar Deb

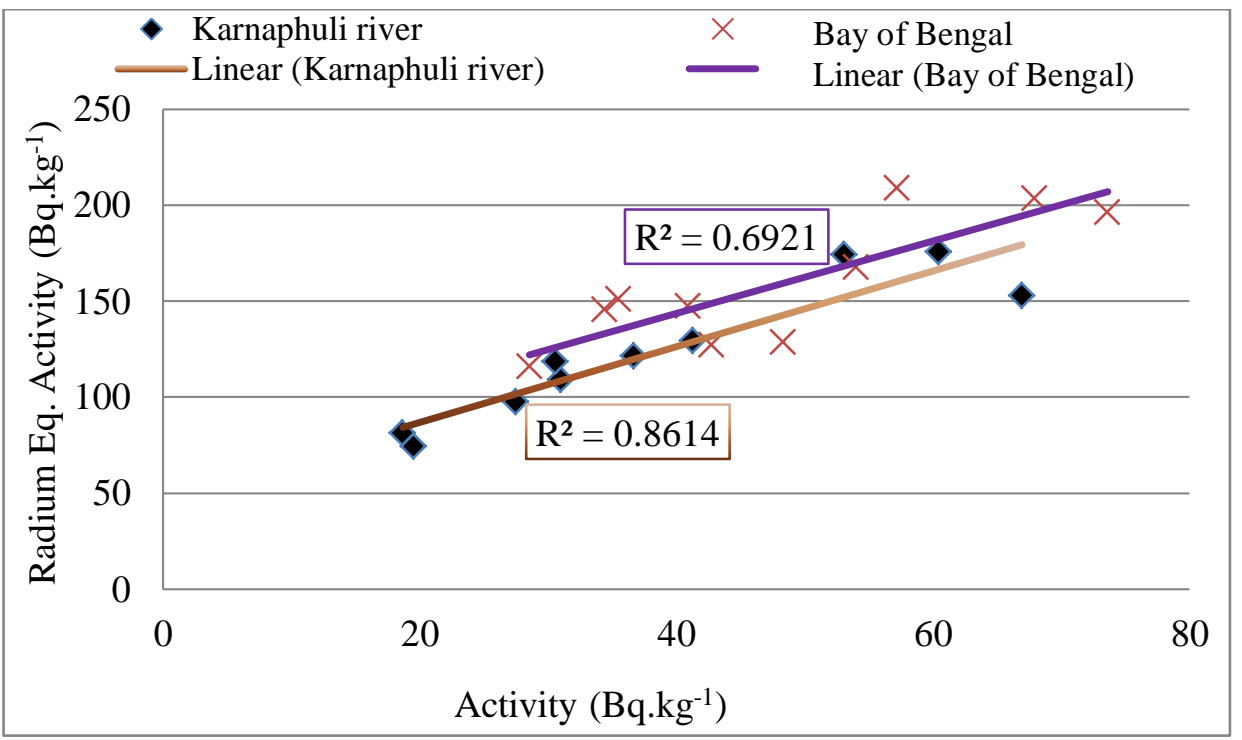

Figure 4: Correlation between $\mathrm{Ra}_{\mathrm{eq}}$ and ${ }^{226} \mathrm{Ra}$ for river and sea samples.

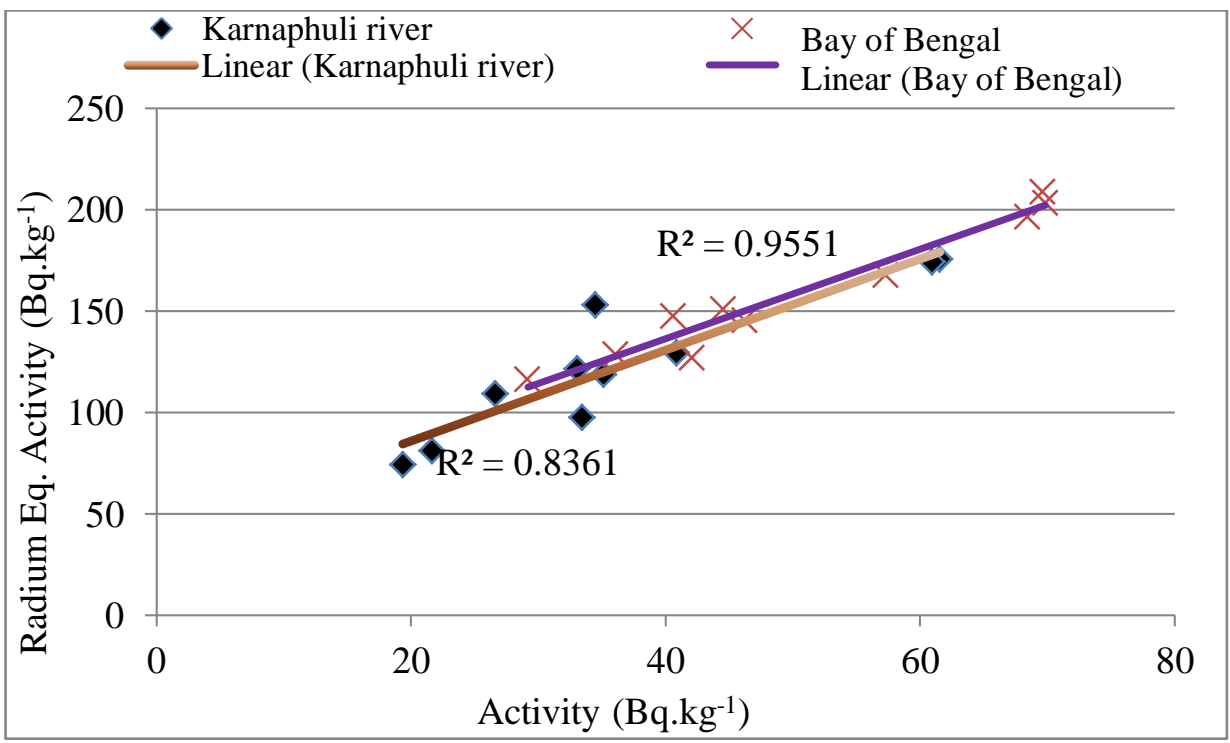

Figure 5: Correlation between $\mathrm{Ra}_{\mathrm{eq}}$ and ${ }^{232} \mathrm{Th}$ in river and sea samples. 


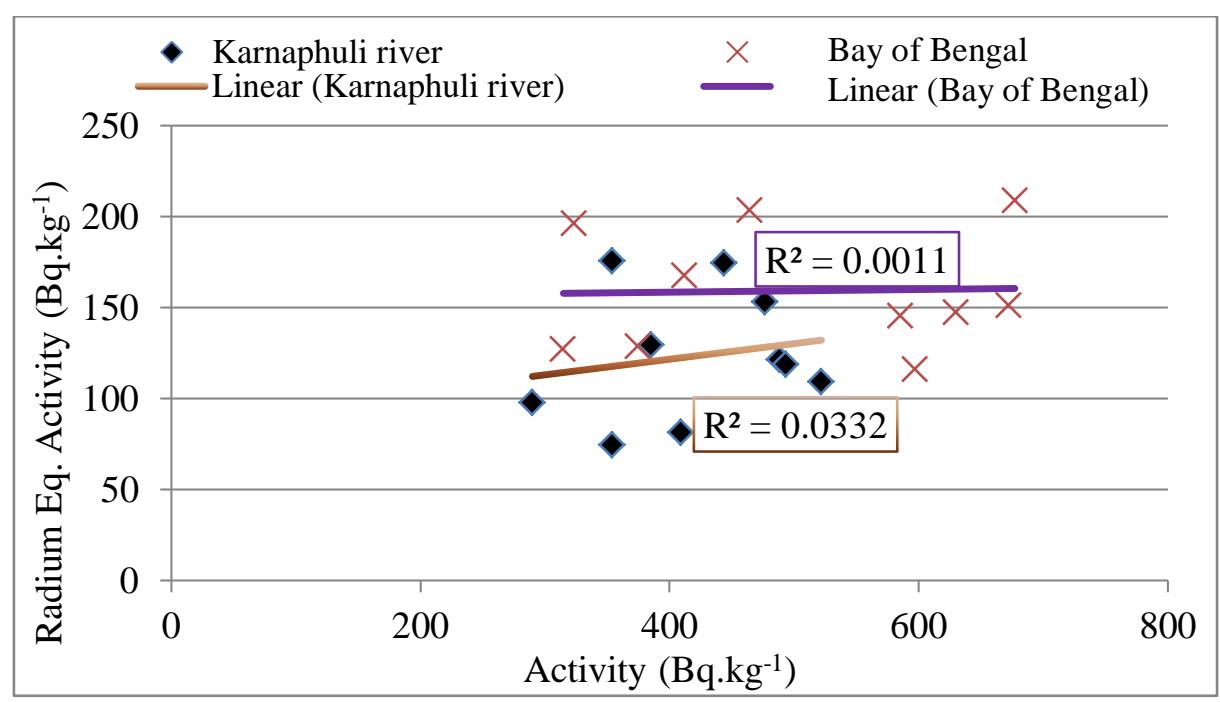

Figure 6: Correlation between $\mathrm{Ra}_{\mathrm{eq}}$ and ${ }^{40} \mathrm{~K}$ for river and sea samples.

\subsection{Chi-Square per Degree of Freedom $\left(\chi_{R}^{2}\right)$}

The values of Chi-square per degree of freedom $\left(\chi_{R}^{2}\right)$ for ${ }^{226} \mathrm{Ra},{ }^{232} \mathrm{Th}$ and ${ }^{40} \mathrm{~K}$ were 70.24, 77.17 and 1.87 respectively for the Karnaphuli River samples and 38.09, 62.02 and 2.06 respectively for the Bay of Bengal samples. The low $\chi_{R}^{2}$ values for ${ }^{40} \mathrm{~K}$ in both the study areas indicate the distributions of ${ }^{40} \mathrm{~K}$ concentration were nearly uniform. The higher $\chi_{R}^{2}$ values for ${ }^{232} \mathrm{Th}$ indicate a wide distribution of ${ }^{232} \mathrm{Th}$ activity concentrations. However, the ${ }^{226}$ Ra activity was more widely distributed in the Karnaphuli River than that of the Bay of Bengal. 
Hasan Murad, Md. Kowsar Alam, Shyamal Ranjan Chakraborty, A. K. M. Rezaur Rahman, S. I. Bhuian, Masud Kamal, Akhi Das Gupta, and Arun Kumar Deb

\section{Conclusions}

In the present work, the following key points are drawn: (1) The mean activity concentrations of ${ }^{226} \mathrm{Ra},{ }^{232} \mathrm{Th}$ and ${ }^{40} \mathrm{~K}$ in sediment of the Karnaphuli River are lower than that of the Bay of Bengal, (2) the mean activity concentration of ${ }^{232} \mathrm{Th}$ and ${ }^{40} \mathrm{~K}$ are lower but ${ }^{226} \mathrm{Ra}$ is higher than that of the world average in sediment of the Karnaphuli River, (3) the mean activity concentrations of ${ }^{226} \mathrm{Ra},{ }^{232} \mathrm{Th}$ and ${ }^{40} \mathrm{~K}$ in the Bay of Bengal are higher than the world average value, (4) the obtained correlations are positive and the $\chi_{R}^{2}$ values indicate that the distribution is within the safety limit and (5) the obtained values of external and internal radiation hazard indices are less than that of the permissible value 1 [14]. So, on the basis of values obtained, it is concluded that no harmful radiation effects are exposed to the public going to the beaches and river sides for recreation or to the sailors and fishermen involved in their activities in the area as a result of the activity of coastal sediments. Hence, no radioactive threat is obtained in the costal belts linked into the Pacific Ocean through the Bay of Bengal of Chittagong city, Bangladesh due to the nuclear reactor accident at Fukushima, Japan.

\section{References}

[1] UNSCEAR: "United Nations Scientific Committee On The Effects Of Atomic Radiation, UNSCEAR Report to the General Assembly with Scientific Annexes", United Nations, New York, 2000, 1 p. 1.

[2] Hasan M. Khan, Khalid Khan M. A. Atta and Farooq Jan: Journal of the Chemical Society of Pakistan, 1994, 16(3), 183. 
[3] A. Sroor, S. M. El-Bahi, F. Ahmed and A. S. Abdel-Haleem: Applied Radiation and Isotopes, 2001, 55(6), 873, doi: 10.1016/S09698043(01)00123-3

[4] P. Chiozzi V. Pasquale, and M. Verdoya: Radiation Measurements, 2002, 35(2), 147. doi: 10.1016/S1350-4487(01)00288-8.

[5] A. Amekudzie, G. Emi-Reynolds, A. Faanu, E. O. Darko, A. R. Awudu, O. Adukpo, L. A. N. Quaye, R. Kpordzro, B. Agyemang and A. Ibrahim: World Applied Sciences Journal, 2011, 13(11), 2338.

[6] William R. Leo: “Techniques for Nuclear and Particle Physics Experiments”, Second Edition, Springer-Verlag, Germany 1994.

[7] International Atomic Energy Agency (IAEA), "Extent of Environmental Contamination by Naturally Occurring Radioactive Material (NORM) and Technological Options for Mitigation, IAEA”, IAEA Technical Reports 2003, 419, 7 .

[8] Analytical Quality Control Services (AQCS): “Intercomparison Runs Reference Materials, IAEA AQCS Catalogue for Reference Materials and Intercomparison Exercises 1998/1999”, IAEA AQCS Catalogue 1998 \& 1999.

[9] K. Debertin and R. G. Helmer: "Gamma and X-ray Spectrometry with Semiconductor Detectors", North-Holland Publishers, Amsterdam, 1988.

[10] A. El-Taher: Radiation Protection Dosimetry, 2010, 138(2), 166. doi: $10.1093 / \mathrm{rpd} / \mathrm{ncp} 205$ 
134 Hasan Murad, Md. Kowsar Alam, Shyamal Ranjan Chakraborty, A. K. M. Rezaur Rahman, S. I. Bhuian, Masud Kamal, Akhi Das Gupta, and Arun Kumar Deb

[11] J. P. Bolivar, R. Garcia-Tenoria, M. Garcia-Leon: IAEA International Nuclear Information System (INIS), IAEA Reference Number 27047249, 1994, 27(14), 134.

[12] M. K. Alam, S. R. Chakraborty, A. K. M. Rezaur Rahman, A. K. Deb, M. Kamal, M. I. Chowdhury, M. S. Uddin: Radiation Protection Dosimetry, 2013, 153(3), 316. doi:10.1093/rpd/ncs117

[13] T. Jabbar, K. Khan, M. S. Subhani, P. Akhter, A. Jabbar: Radiation Protection Dosimetry, 2008, 132(1), 88. doi: 10.1093/rpd/ncn253

[14] UNSCEAR: "United Nations Scientific Committee on the Effects of Atomic Radiation, Exposure from Natural Sources of Radiation: UNSCEAR Report to the General Assembly, with annexes"; United Nations, New York, 1988.

[15] Norman H. Custall, Ingvar L. Larsen, and Curtis R. Olsen: Nuclear Instruments and Methods in Physics Research, 1983, 206(1-2), 309. doi: 10.1016/0167-5087(83)91273-5

[16] J. Beretka and P. J. Mathew: Health Physics, 1985, 48(1), 87. doi: 10.1097/00004032-198501000-00007

[17] Danilo C. Vasconcelos, Arno H. Oliveira, Mário R. S. Silva, Rodrigo Penna, Talita O. Santos, Claubia Pereira, Zildete Rocha, and Maria Ângela B. C. Menezes: IAEA International Nuclear Information System (INIS), 2009, 41(39), IAEA Reference Number 41109077.

[18] W. R. Alharbi: Life Science Journal, 2012, 9(4), 3573. Available at http://www.lifesciencesite.com 
[19] M. Bolca, M. M. Saç, B. Çokuysal, T .Karalı, and E. Ekdal: Radiation Measurements, 2007, 42(2), 263. doi: 10.1016/j.radmeas.2006.12.001

[20] Isaac R. Ajayi: Medwell Journals: Research Journal of Applied Sciences, 2008, 3(3), 183.

Available at http://medwelljournals.com/abstract/?doi=rjasci.2008.183.188

[21] Xinwei Lu, Xiaolan Zhang and Fengling Wang: Environmental Geology, 2008, 53(7), 1475. doi: 10.1007/s00254-007-0756-0

[22] A. El-Taher, and H. M. Madkour: Indian Journal of Geo-Marine Sciences, 2013, 42(1), 35.

Available at http://nopr.niscair.res.in/handle/123456789/15983

[23] M. A. M. Uosif: Radiation Protection Dosimetry, 2007, 123(2), 215. doi: 10.1093/rpd/ncl103

[24] S. U. El-Kameesy, S. Abd El-Ghany, S. M. El-Minyawi, Z. Miligy and E. M. El-Mabrouk: Turkish Journal of Engineering and Environmental Sciences, 2008, 32(4), 245. (Available at

http://journals.tubitak.gov.tr/engineering/issues/muh-08-32-4/muh-32-4-70804-3.pdf)

[25] N. M. Fahmi, A. El-Khatib, Y. M. Abd El-Salam, M. H. Shalaby, M. M. ElGally and M. A. Naim: "Proceedings of Tenth Radiation Physics \& Protection Conference”, during 27-30 November, 2010, Nasr City - Cairo, Egypt, 2010, EG1100494, 391. (Available at http://www.iaea.org/inis/collection/NCLCollectionStore/_Public/42/076/4207 6665.pdf) 
136 Hasan Murad, Md. Kowsar Alam, Shyamal Ranjan Chakraborty, A. K. M. Rezaur Rahman, S. I. Bhuian, Masud Kamal, Akhi Das Gupta, and Arun Kumar Deb

[26] Prasong Kessaratikoon, Supphawut Benjakul and Suchin Udomsomporn: Kasetsart Journal (Natural Science), 2007, 41(5), 157. (Available at http://thaiagris.lib.ku.ac.th/eng/)

[27] Khondaker Mohammed Nazrul Islam, Debasish Paul, Md. Mahbubur Rahman Bhuiyan, Amina Akter, Budrun Neher and Sheikh Mohammad Azharul Islam: Journal of Environmental Protection, 2012, 3(9), 1078. doi: 10.4236/jep.2012.39126

[28] H. Lawluvi, E. O. Darko, C. Schandorf, A. Fannu, A. R. Awudu and D. O. Kpeglo: Research Journal of Environmental and Earth Sciences, 2011, 3(6), 729. (Available at http://maxwellsci.com/print/rjees/v3-729-736.pdf)

Manuscript received on 07 August, 2017, Revised manuscript received on 17 July, 2018 and accepted on 17 July, 2018

The Chittagong Univ. J. Sc. Vol. 40, 2018 